\title{
Decisional issues in antibiotic prescribing in French nursing homes: An ethnographic study
}

\author{
Taghrid Chaaban, ${ }^{1-3}$ Mathieu Ahouah, ${ }^{1}$ Pierre Lombrail, ${ }^{1}$ Héléne Le Febvre, ${ }^{2}$ Adnan Mourad, ${ }^{3}$ \\ Jean-Manuel Morvillers, ${ }^{1}$ Monique Rothan-Tondeur ${ }^{1}$
}

\author{
${ }^{1}$ Nursing Sciences Research, LEPS, University Paris 13, Sorbonne, Paris, France; ${ }^{2}$ Faculty of Nursing \\ Sciences, Montréal University, Centre-Ville, Montréal, Canada; ${ }^{3}$ Islamic University of Lebanon (IUL), \\ Faculty of Public Health, Beirut, Lebanon
}

\begin{abstract}
Significance for public health
The study described in this paper is significant for the field of public health for several reasons. The consumption of antibiotics is a serious worldwide problem. Before implementing an antibiotic stewardship in $\mathrm{NH}$, we needed to better know how antibiotic were prescribed. The knowledge from this study should be used in practice to optimize the role of nurses in reducing inappropriate prescribing of antibiotics. The efficient interaction of nurses during the decision-making process of the antibiotic prescribing produce an appropriate care in $\mathrm{NH}$, which would be contributed to decrease the microbial resistance and therefore the cost of health services.
\end{abstract}

\begin{abstract}
Background: Medication prescription is generally the responsibility of doctors. In nursing homes, the nursing staff is often the first to suspect an infection. Today, physicians are more confident with nursing assessment, relying primarily on nursing staff information. Very few studies have investigated the nurses' influence on decision of medical prescription. This study investigates the role of nurses in antibiotic prescribing for the treatment of suspected infections in nursing home residents.

Design and methods: An ethnographic study based on semi-structured interviews and participant observations was conducted. Sixteen nurses and five doctors working in five nursing homes in Paris, France participated between October 2015 and January 2016.

Results: Given their proximity to elderly residents, registered nurses at the nursing homes occasionally assisted doctors in their medical diagnostic. However, nurses who are theoretically incompetent have met difficulties in their ability to participate in their decisions to prescribe antibiotics when managing residents' infections.

Conclusion: if proximity and nursing skills reinforce the relevance of the clinical judgment of nurses, the effective and collaborative communication between the nurse and the doctor may help the nurse to enhance their role in the antibiotic prescribing in nursing homes, which would enhance antimicrobial stewardship efficiency.
\end{abstract}

\section{Introduction}

Aging has been shown to be associated with biological frailty. ${ }^{1}$ In view of this fact, infections not only constitute a major public health problem worldwide, but also account for significant morbidity and mortality in elderly populations, particularly the residents in nursing homes (NHs). ${ }^{2,3}$ Seniors in NHs are prone to infections, notably those affecting the urinary and respiratory tract. ${ }^{4,5}$ As physicians are not always present in NHs, the nursing staff is often the first to suspect an infection and report it to the physicians; therefore, nursing staff could have a major impact on physicians' decisions regarding the treatment of the residents in nursing homes. Nursing staff are adopting significant roles in infection management; thus, they play a fundamental role in NHs through their continuous attendance and the skills they may possess. Today, physicians are more confident with nursing assessment, relying primarily on nursing staff information to guide their decisions to prescribe antibiotics. ${ }^{6}$

Attending physicians at NHs often prescribe antibiotics (ATBs) to cure infections among the senior residents. ${ }^{7}$ Several factors such as resident's clinical situation and its personal and family history may influence this prescription decision..$^{3,8,9}$ In fact, inappropriate use of ATBs has been reported in similar contexts. ${ }^{7}$ Further, ATB consumption without indication is yet another public health concern that needs to be addressed knowing the potential for development of antimicrobial resistance and its associated health care costs. ${ }^{10}$

In some countries, like the United States and New Zealand, nurse practitioners, that have had additional training courses, are often given the role of prescribing ATBs. ${ }^{11}$ Meanwhile, this prescription act is solely the responsibility of the attending physician in many other countries, ${ }^{3}$ such as France. Nurses are ATB first responders, principal communicators, and monitors of response to ATB treatment. The central role of nurses in routine patient care makes it clear that they perform numerous functions that are critical to the successful antimicrobial stewardship process performance. They are also the primary source of information for patients and their families about the prescribed ATBs and their appropriate use. ${ }^{12}$ Indeed, the nursing staff not only provides an assessment of the patient's clinical condition to the doctor, ${ }^{13}$ but also supports doctors in safe prescribing and, hence, reduces medication errors. ${ }^{14}$ This combined interaction between both of these professions in terms of knowledge and skills can influence patient care. ${ }^{15}$ One study conducted by Lim CJ et al. ${ }^{6}$ has even gone further in describing this inter-professional interaction between nurses and physicians. It has pointed out that general practitioners rely solely on the assessment of nurses in NHs in the management of certain infections.

This paper aims to investigate the nurse's role in ATB prescribing decisions for the treatment of suspected infections in $\mathrm{NH}$ residents, as well as to understand how the inter-professional collaboration between nursing staff and prescribing doctors is developed in such context. 


\section{Materials and Methods}

\section{Study design}

This is a qualitative study design based on an ethnographic approach, as described by Beaud and Weber in 2010. This approach is grounded in the field of social research and aims to explore and understand complex social phenomena like nurse's role in ATB prescribing in nursing homes. It included problem-definition, making contact, entering the field, establishing and maintaining a role in the field, collecting first hand data, reporting data, exiting from the field and, finally, analysis and theoretical processing.

\section{Sample selection and sampling plan}

Five NHs were randomly selected for this study from the two larger groups of Parisian NHs, ORPEA and KORIAN. Purposive sampling was used for the selection of sample members who will best contribute to the informational needs of the study. Participants were chosen based on their work schedule and their availability during our observation days. A total of sixteen nurses (4 coordinating nurses and 12 registered nurses responsible for implementation of drug prescription orders) and five doctors ( 3 coordinating and 2 prescribing), working in the selected NHs, were included in this study.

\section{Data collection tool}

Participant observations, together with the semi-structured interviews, were conducted between October 2015 and January 2016. These two main methods of data production were used to provide an ethnographic explanation of the nurse's role in ATB prescribing. ${ }^{16} \mathrm{~A}$ total of approximately 120 hours of participant observations were completed. Observational field notes of informal conversations of health providers, ${ }^{17}$ as well as methodological notes on the institutional protocols, daily activities, physicians' schedules, health workers' environment, presence of a pharmacy and drugs in the nurses' office, etc.... were taken by the researchers. The registered nurses were followed up from the moment of suspicion of an infection until the application of the prescribed ATB. Comprehensive observation reports were written within two days after the observation in each NH. In addition, the study participants were interviewed at their workplace by the first author. The semi-structured interviews were digitally audiorecorded, as well as transcribed verbatim by the first author and were checked for accuracy by an independent transcriber. The major ethical considerations involved in data collection process were respected in this study. The second author negotiated the directors of NHs and gained approval to access them. A written informed consent was obtained from all study participants. The data were collected anonymously to protect privacy and maintain confidentiality. The research was approved by steering committee.

\section{Data analysis}

The qualitative data collected were analyzed using inductive content analysis method to identify themes or patterns within data. ${ }^{18}$ The analysis of verbatim transcripts of the interviews and detailed field notes was performed in several steps. First, each member of the research team carefully read over each transcript and field note to obtain an overall understanding of the participant's points of view. The qualitative analysis of interviews and field notes was supported by NVivo software (version 11). Phrases that portrayed key thoughts or concepts were highlighted, and similar phrases were grouped to identify the units of analysis. These text units were coded using words or phrases related to the aim of this study. The various codes were compared based on the differences and similarities and then sorted into two categories to facilitate reporting final results. These categorizations were continually reviewed and discussed during weekly meetings within the research group until an agreement was reached to ensure their appropriateness.

\section{Findings}

A total of 21 transcripts were analyzed. The average age of the study participants was 38.1 years $(\mathrm{SD}=13.8)$, and the majority $(76.2 \%)$ were females (Table 1$)$. Six prescriptions of ATBs were observed during the process of data collection. This study revealed several interesting findings. Through direct observation and informal interviews, it was noted that nurses at the NHs were very familiar with the elderly residents. They had to be on-site for at least five days a week throughout the year to assess the residents' clinical condition when symptoms arise, and quickly begin their initial treatment. This quality was based on the notion of 'proximity'. The fact that nurses are close to residents in the sense that they take care of them on a daily basis, implies sharing different moments during each day. Thanks to this proximity, the caregiver can appreciate at best and on a daily basis the general condition of the resident. This allows for greater acuity in the development of clinical judgment. This obviously influences the relationships with other stakeholders and ultimately in the medical decision. Moreover, the study showed a range of aspects for the interaction between nurses and physicians, starting from the exemption of the nurse from the medical decision, to the possible collaboration between the doctor and the nurse based on competence and expertise of the latter. The term 'skill' was used to define this quality. Indeed, the nurse's role in ATB prescription for the treatment of suspected infections in NH residents is defined on the basis of 'proximity', 'theoretical knowledge' and 'skills'.

\section{Proximity}

This study showed that all participating nurses worked closely with $\mathrm{NH}$ residents, more often than physicians. Nurses at the NHs were familiar with the medical histories and life stories of the elderly residents for whom they took care sometimes for many years. In this study, the nurse was found to accurately update and summarize relevant clinical information about the NH resident and transmits these figures to the doctor. All prescribing doctors $(2 / 2)$ stated that they visited the $\mathrm{NH}$ residents systematically in the presence of their caring nurse.

"We are very close to NH residents; we see them more often than physicians." [NH4-Nurs1]

"We know our NH residents; we often know how they react when they have a urinary tract infection (UTI)." [NH1-Nurs2]

"Nurses are familiar with the life story of the residents." [NH5observation]

"The prescribing doctor visits our NH residents every Tuesday." [NH1-observation]

\section{Theoretical knowledge}

This study pointed out the inadequate knowledge that some nurses had on the proper use of ATBs. Only 14\% of all study participants stated that they received specific training on ATB therapy. The lack of such theoretical knowledge had greatly influenced nurses' ability to interact with the physician during the ATB prescription process. The prescribing doctors $(2 / 2)$ reported that nurses in France lacked necessary knowledge to discuss the prescription of ATBs with the prescriber. And so, they were not able to play a role in the decision-making regarding ATB use. 
"We were never provided training on the proper use of ATBs." [NH1-Nurs-Co]

"A nurse in France does not have the necessary clinical competencies regarding ATB prescribing: I do not know how he/she will discuss the matter with the doctor; I do not think it is his/her role.'[NH4-Presc]

Some nurses (4/16) said that, through giving proper education on ATB therapy, nurses might become more aware and able to discuss prescription of ATBs with prescribers.

"To say that ATBs are useless is not very professional. Therefore, we must begin by stating arguments... that is why I think that the implementation of a training program on ATB therapy in $\mathrm{NH}$ would really be more than wise. It will help us discuss the prescription with the doctor." [NH5- Nurs-Co]

\section{Skills}

In this case, nursing skills can be considered to lie in several categories.

\section{Technical skills}

As prescribing doctors are not present in NHs on a daily basis, they are not the first actors to respond in case of an emergency as in the case of hyperthermia, dyspnea, disturbance of consciousness, change of behavior.... The study results indicated that the interviewed nurses acquired skills in handling clinical and para-clinical evaluations and in responding to emergencies.

"With worsening of the general condition, the person loses his habits, does not get up, does not eat..." [NH2-Nurs2]

"The nurse takes the resident's temperature, tests his urine and gives him an antipyretic..." [NH1-observation]

"... A urine dipstick, if we see that it is positive, we ask the treating doctor, once we receive a urine culture prescription by fax, we perform the culture" [NH2- Nurs-Co]

All nurses reported that their unique role consisted of providing drugs in conformity with the doctors' orders. vation]

"The physician prescribes and the nurse applies." [NH5-obser-

Therefore, the majority of respondents reported that they monitor the regression of signs of infection and test results to assess the effectiveness of antibiotic therapy.

"Symptom regression is the most sought-after sign. It is rare that biology is controlled"[NH2- Nurs2]

\section{Reasoning skills}

Some nurses in this study (6/16) described how they used their reasoning skills to assist doctors in making a sound clinical judgment and to recommend ATBs prescription based on the resident's clinical signs and symptoms.

"There are doctors who ask us what we think, so we talk about it and then we come to a conclusion..." [NH3-Nurs2]

"The nurse recommends ATB prescribing, stating that the resident has a cough and fever" [NH4-observation]

\section{Communication skills}

Another interesting finding, which was identified as central in our study, is the value of communication. This relational skill refers to the inter-professional collaboration. Such interaction between nurses and doctors at the NHs could be described as social and friendly, and the conversations that took place were light and rather superficial. In this study, the nurse was found to accurately update and summarize relevant clinical information about the $\mathrm{NH}$ resident and transmits these figures to the doctor.

"Based on clinical observation, I will turn to the doctor." [NH3-Nurs-Co]

Table 1. Characteristics of participants.

\begin{tabular}{|c|c|c|c|c|c|c|c|}
\hline No. Interview & Position & Age & Gender & $\begin{array}{l}\text { Years of } \\
\text { experience } \\
\text { in the } \mathrm{NH}\end{array}$ & $\begin{array}{l}\text { Years of } \\
\text { experience out } \\
\text { of the NH }\end{array}$ & $\begin{array}{l}\text { Duration of } \\
\text { interview in } \\
\text { minutes }\end{array}$ & $\begin{array}{l}\text { Specific training } \\
\text { on antibiotherapy }\end{array}$ \\
\hline 1 & Nurse Coordinator & 53 & $\mathrm{~F}$ & 18 & 15 & 14 & No \\
\hline 2 & Nurse & 28 & $\mathrm{~F}$ & $2 y \& 3 m$ & - & 10 & No \\
\hline 3 & Nurse & 34 & M & 10 & - & 15 & No \\
\hline 4 & Nurse & 23 & $\mathrm{~F}$ & 2 & - & 15 & No \\
\hline 5 & Nurse & 66 & $\mathrm{~F}$ & 30 & 10 & 10 & No \\
\hline 6 & Nurse Coordinator & 29 & $\mathrm{~F}$ & 1 & 6 & 10 & No \\
\hline 7 & Nurse & 28 & $\mathrm{~F}$ & $1 \mathrm{y} \& 6 \mathrm{~m}$ & 3 & 15 & No \\
\hline 8 & Nurse & 30 & M & 2 & - & 10 & No \\
\hline 9 & NH doctor Coordinator & 46 & $\mathrm{~F}$ & $1 \mathrm{y} \& 6 \mathrm{~m}$ & 15 & 15 & Yes \\
\hline 10 & Prescriber & 60 & $\mathrm{~F}$ & 15 & 30 & 16 & Yes \\
\hline 11 & Nurse Coordinator & 27 & $\mathrm{~F}$ & 1 & 6 & 16 & No \\
\hline 12 & Nurse & 25 & $\mathrm{~F}$ & $2 y \& 6 m$ & - & 13 & No \\
\hline 13 & Nurse & 28 & M & 2 & - & 20 & No \\
\hline 14 & Prescriber & 55 & M & 12 & 30 & 10 & No \\
\hline 15 & Nurse & 32 & $\mathrm{~F}$ & $2 y \& 5 m$ & 2 & 15 & No \\
\hline 16 & Nurse & 36 & $\mathrm{~F}$ & 12 & - & 10 & No \\
\hline 17 & NH Doctor Coordinator & 46 & $\mathrm{~F}$ & 6 & 10 & 10 & Yes \\
\hline 18 & Nurse Coordinator & 52 & $\mathrm{~F}$ & $2 \mathrm{~m}$ & 15 & 10 & No \\
\hline 19 & Nurse & 24 & $\mathrm{~F}$ & 3 & - & 10 & No \\
\hline 20 & Nurse & 24 & $\mathrm{~F}$ & 2 & 1 & 10 & No \\
\hline 21 & NH doctor Coordinator & 56 & $\mathrm{M}$ & 4 & 16 & 17 & No \\
\hline
\end{tabular}


One participating nurse stated that, when the prescribed ATBs were seen unnecessary, it was essential to follow the medical hierarchy because of nurses' lack of theoretical skills.

"If I find that the prescribed ATB is not necessary, I talk to him (i.e., the doctor)..." [NH1- Nurs4]

However, some nurses (4/16) reported that their professional interaction with doctors were more often about the form of the drugs than whether to describe an ATB. Discussing ATB prescription with the prescribing physicians was clearly challenging and rather difficult for some nurses (3/16).

"We can give our opinion, and after that, depending on the doctor, we intervene or not... these conversations are obviously more about the form of the drug than the prescription decision that is left to the doctor." [NH1-Nurs4]

The participating nurses (5/16) admitted that doctors felt confident in their skills and their ability to assume responsibilities so they encouraged the nurses to participate in discussions about the prescribed ATBs.

"We discuss these issues with the doctor, doctors trust us enormously."[NH2- Nurs-Co]

"Doctors often ask: Do you think this patient doesn't go well without this ATB? What was his previous ATB...?" [NH1-observation]

\section{Discussion}

A nurse is the first point of contact for patients in the NH setting. Nurses play a pivotal role in the management of suspected infections due to their "proximity" ${ }^{19,20}$ They are often the first health care professionals to detect any change in NH resident's behavior critical to health, and to identify emergency conditions that necessitate the request of medical assistance. Due to their infrequent physicians' visits to $\mathrm{NH}$, the nurses assist them to detect signs of infection for elderly patients. ${ }^{3}$ The professional who attends to residents on a daily basis is more likely to understand their different personalities, values and particularities. ${ }^{21}$ In a study conducted by Funkesson et al., ${ }^{22}$ the author shows that the experience and knowledge of nurses together with how physically close to the elderly they work are essential factors that affect their clinical reasoning.

Although the nurses participating in our study do not have diagnostic procedures to confirm a respiratory tract infection, they can perform a urinalysis dipstick test as a first form of preclinical diagnoses in case of suspected UTI. These findings are very similar to those reported in the two studies conducted by Schweizer et $a l .{ }^{20}$ and Ribeiro, ${ }^{23}$ which show that the nurse can assist in the diagnostic process of UTIs. The nurses in these studies have performed urinalysis using a dipstick as their first tool in the diagnosis of UTIs. Moreover, Schweizer and her colleagues ${ }^{20}$ shows that the nurses seldom contacted a doctor immediately after noticing possible symptoms associated with UTIs in elderly NH residents.

Some respondents have felt that the majority of ATB prescriptions in the NHs are not always necessary. Similarly, prospective studies have shown that almost half of the courses of antibiotics prescribed in NHs are useless. ${ }^{24}$ This study result comes in consistency with the findings of some previous studies that described the reasons for unnecessary prescriptions, such as the uncertainty of assigning diagnoses and fear of failure. ${ }^{6,8,25}$ Indeed, preventive prescriptions are frequently observed in poly-pathological patients and in the absence of biological tests confirming the diagnosis. Due to infrequent physicians' visits to $\mathrm{NH}$, often owing to time constraints, ATB prescriptions 'over the phone' are sometimes issued for the elderly patients who do not always automatically develop fever in response to infection and are often unable to communicate their symptoms. ${ }^{3}$

Our study has also indicated that nurses lack adequate knowledge about the proper use of ATBs. This result is similar to that reported in the two studies conducted by Edwards et al. ${ }^{26}$ and Lim CJ et al., ${ }^{6}$ who have demonstrated that nurses are not trained on the proper use of ATBs. The nurses in our study are particularly motivated to participate in an educational training program on ATB therapy, which they believe it will enable them to engage in discussions about ATBs prescription with the prescribing physician. According to Gillepsie et al., ${ }^{27}$ the involvement of nurses in an ATB education program, that focuses on evaluating the safety and appropriateness of ATB therapy on patients, is particularly important for their contribution in ATB prescribing decisions and has actually led to an increase in nurses' antimicrobial management knowledge. This educational approach will be an asset in the healthcare industry to lower the incidence of unnecessary ATB administration and its unwanted costs. ${ }^{12}$

Based on the findings of this study, the role of nurses in the inter-professional communication is seen as limited to social relationships. This can be attributed to the lack of thorough knowledge, which, when grasped, helps nurses assert themselves, gain confidence in their abilities, and enhance their contribution to professional communication. The acknowledgement is a power in the relationships, caring that nurses provide and sharing with the physician the decision-making process. ${ }^{28}$

It appears based on the results of various interviews that the nurses participating in this study have expressed difficulty in discussing ATB prescription with prescribers and their role is limited to the implementation of the prescription. Such results are different from those revealed in an article by Jutel \& Menkes, ${ }^{29}$ who notes that most nurses have discussed the treatment options with the prescribing physician, and, according to another study conducted outside of Europe, the majority of nurses are involved in the decisionmaking regarding prescription drugs. Similarly, Castledine has reported that senior nurses can influence prescribing medical decisions and reduce prescription errors involving medication dosage or incorrect prescribed drug for a patient. ${ }^{14}$

Some participants nurses stated they thought doctors trusted them to participate in the prescribing decision but this is not supported by any evidence from doctors the study results by Lim CJ et $a l .{ }^{6}$ have pointed out those doctors generally rely on the clinical assessment of skilled nurses in NHs for the management of certain infections. As nurses are responsible for a substantial proportion of decisions and judgments in the healthcare system, their acquisition of practical as well as reasoning skills is critically important for their competence. ${ }^{30}$

Overall, it is worth mention that the desire for inter-professional collaboration in this study is more frequently expressed by nurses than doctors. Several studies in different countries have demonstrated the presence of a higher level of demand among nurses and a greater willingness to collaborate throughout the prescribing process. ${ }^{31,32}$ Indeed, effective and collaborative communication between nurses and doctors remains a paramount goal for the development of an optimal care and treatment plan. ${ }^{28,33}$

\section{Strengths and limitations}

While a few old studies on the role of nurses in French NHs do exist, research that addresses their place in medical or ATB prescription is generally lacking. One limitation of this study might be that only five NHs were observed during a relatively short time period. Therefore, events and behaviors that occur infrequently could have been missed. The key issue in this qualitative ethnographic study was to reflect the participants' opinions and not to 
seek sample representativeness or generalizability of the findings neither in the five NHs in Paris or elsewhere in France nor internationally. Although it was not the intent of this study to make its results generalizable, it obviously provided important and valuable information about the role of nurses in ATB prescription in NH settings. Lastly, the results of this study may be limited by sample size and setting (five NHs), but the use appropriate methodology, rigors of data collection and data analysis provided a range of approaches to improve the validity and quality of the findings, all of which enriched the study.

\section{Conclusions}

Nurses have a critical role in the provision of care for elderly NH residents with suspected infections due to their "proximity". Nurses who are theoretically incompetent have met difficulties in their ability to participate in ATB prescribing decisions, but they are more successful in communicating their sound clinical judgment when they are skilled. That is to say; if proximity and nursing skills reinforce the relevance of the clinical judgment of nurses, the lack of effective and collaborative communication between the nurse and the doctor may have a negative impact on the appropriateness of medical prescribing. Indeed, it seems that inter-professional interaction between the nurse and the prescriber may reduce, or even avoid, unnecessary prescription of ATBs.

Work is required in developing templates for use by nursing staff to assist in antimicrobial stewardship initiatives appropriate to the NH residents. Further research should investigate the effects of these interventions on the nursing role in reducing inappropriate antibiotic prescription.

Correspondence: Taghrid Chaaban, University Paris 13, Sorbonne Paris Cite, Nursing Sciences Research chair, Laboratory Educations and Health Practices (LEPS), (EA 3412), UFR SMBH, F-93017, Bobigny, France; Islamic University of Lebanon (IUL), Faculty of Public Health, Beirut, Lebanon.

Tel.: +961.3868597 - Fax: +961.5807719

E-mail: tagochaaban@hotmail.com

Key words: Antibiotic, decision making, elderly, ethnographic.

Contributions: Concept and design: TC, MA, JM, MRT; Drafting work:

TC, MA, AM; Critical revising: PL, HL, JM, MRT; Final approved to publication: PL, HL, AM, JM, MRT; all authors agreed to be accountable.

Conflict of interest: the authors declare no potential conflict of interest. Acknowledgements: We would like to acknowledge the support of Steering Committee: Cartier T., Le Gonidec; Gavazzi G; P., Zamponi DB., Bendelac H., Tribalat R., Bertin-Hugault F., Armaingaud D. Funding: this research received funding from the Alere Society, Réunica Ag2R, and Fondation Caisse d'Epargne, IUL.

Conflict of interests: the authors declare no conflict of interests.

Received for publication:14 December 2018.

Revision received: 20 August 2019.

Accepted for publication: 30 August 2019.

oCopyright: the Author(s), 2019

Licensee PAGEPress, Italy

Journal of Public Health Research 2019;8:1533

doi:10.4081/jphr.2019.1533

This work is licensed under a Creative Commons Attribution NonCommercial 4.0 License (CC BY-NC 4.0).

\section{References}

1. Leung E, Weil DE, Raviglione M, Nakatani H. and on behalf of the World Health Organization World Health Day Antimicrobial Resistance Technical Working Group. The WHO policy package to combat antimicrobial resistance. Bull World Health Organ 2011;89:390-2.

2. Rothan-Tondeur M, Piette F, Lejeune B, et al. Infections in nursing homes: is it time to revise the McGeer criteria? J Am Geriatr Soc 2010;58:199-201.

3. Fleming A, Bradley C, Cullinan S, Byrne S. Antibiotic prescribing in long-term care facilities: a qualitative, multidisciplinary investigation. BMJ Open 2014;4:e006442.

4. Benoit SR, Nsa W, Richards CL, et al. Factors associated with antimicrobial use in nursing homes: A multilevel model. J Am Geriatr Soc 2008;56:2033-44.

5. Chami K, Gavazzi G, Carrat F, et al. Burden of infections among elderly in nursing homes: a cross-sectional cluster nationwide survey. J Hosp Infect 2011;79:254-9.

6. Lim CJ, Kwong MW, Stuart RL, et al. Antibiotic prescribing practice in residential aged care facilities - health care providers' perspectives. Med J Aust 2014;201:98-102.

7. Lim CJ, McLellan SC, Cheng AC, et al. Surveillance of infection burden in residential aged care facilities. Med J Aust 2012;196:327-31.

8. Van Buul LW, van der Steen JT, Doncker SM, et al. Factors influencing antibiotic prescribing in long-term care facilities: a qualitative in-depth study. BMC Geriatr 2014;14:136.

9. Crnich CJ, Jump R, Trautner B, et al. Optimizing Antibiotic Stewardship in Nursing Homes: A Narrative Review and Recommendations for Improvement. Drugs Aging 2015;32:699-716.

10. Woodford HJ, George J. Diagnosis and management of urinary infections in older people. Clin Med 2011;11:80-3.

11. Lim A, North N, Shaw J. Nurse prescribing: the New Zealand context. Nurs Prax N Z 2014;30:18-27.

12. Olans RN, Olans Rd, DeMaria A Jr. The Critical Role of the Staff Nurse in Antimicrobial Stewardship -Unrecognized, but Already There. Clin Infect Dis 2016;62:84-9.

13. Muhrer JC. The importance of the history and physical in diagnosis. Nurse Pract 2014;39:30-5.

14. Castleldine G. Prescribing is not the only issue for nurses and drugs. Br J Nurs 2006;15:836-7.

15. Nelson GA, King ML, Brodine S. Nurse-physician collaboration on medical-surgical units. Medsurg Nurs 2008;17:35.

16. Céfaï D. L'enquête de terrain. Paris: La découverte; 2014.

17. Beaud S., Weber F. Guide de l'enquête de terrain. 4th ed. Paris: La découverte; 2010. (Collection Guides Grands Repères).

18. Le Jeune C. Manuel d'analyse qualitative. 1st ed. Paris; 2014.

19. Walker S, McGeer A, Simor AE, et al. Why are antibiotics prescribed for asymptomatic bacteriuria in institutionalized elderly people? A qualitative study of physicians' and nurses' perceptions. Can Med Assoc J 2000;163:273-7.

20. Schweizer AK, Hughes CM, Macauley DC, O’Neill C. Managing Urinary Tract Infections in Nursing Homes: A Qualitative Assessment. Pharm World Sci 2005;27:159-65.

21. Tanner C. Thinking like a nurse: A research-based model of clinical judgment in nursing. J Nurs Educ 2006;45:204-11.

22. Funkesson KH, Anbäcken EM, Ek AC. Nurses' reasoning process during care planning taking pressure ulcer prevention as an example. A think-aloud study. Int J Nurs Stud 2007;44:1109-19.

23. Ribeiro S. Nursing management of urinary tract infections. Nurs Older People 2015;27:24-9. 
24. Rotjanapan P, Dosa D, Thomas KS. Potentially inappropriate treatment of urinary tract infections in two Rhode Island nursing homes. Arch Intern Med 2011;171:438-43.

25. Hulsher ME, Van Der Meer JW, Grol RP. Antibiotic use: how to improve it? Int J Med Microbiol 2010;300:351-6.

26. Edwards R, Drumright LN, Kiernan M, Holmes A. Covering more Territory to Fight Resistance: Considering Nurses' Role in Antimicrobial Stewardship. J Infect Prev 2011;12:6-10.

27. Gillespie D, Hood K, Bayer A, et al. Antibiotic prescribing and associated diarrhoea: a prospective cohort study of care home residents. Age Ageing 2015;44:853-60.

28. Manojlovich M. Power and Empowerment in Nursing: Looking Backward to Inform the Future. Online J Issues Nurs 2007;12:2.

29. Jutel A, Menkes DB. Nurses' reported influence on the prescription and use of medication. Int Nurs Rev 2010;57:92-7.
30. Tompson C, Cullum N, McCaughan D, et al. Nurses, information use, and clinical decision making: The real world potential for evidence-based decisions in nursing. Evid Based Nurs 2004;7:68-72.

31. Hojat M, Gonnella JS, Nazca TJ, et al. Comparisons of American, Israeli, Italian and Mexican physicians and nurses on the total and factor scores of the Jefferson scale of attitudes toward physician-nurse collaborative relationships. Int J Nurs Stud 2003;40:427-35.

32. Hansson A, Avremo T, Marklund B, et al. Working togetherprimary care doctors' and nurses' attitudes to collaboration. Scand J Publ Health 2010;38:78-85.

33. Narasimhan M, Eisen LA, Mahoney CD, et al. Improving Nurse Physician Communication and Satisfaction in the Intensive Care Unit With a Daily Goals Worksheet. Am J Crit Care 2006;15:217-22. 